\section{Electronic holding relay for control of ac and dc circuits*}

\author{
DAVID O. PETTIJOHN and E. C. T. WALKER \\ Massachusetts Institute of Technology, Cambridge, Massachusetts 02139
}

This relay instantaneously and reliably switches ac and dc loads to either of two states without introducing acoustic or electric noise to associated audio and logic circuitry.

Experiments conducted in our laboratory often measure the latency of Ss' responses to the onset or occurrence of a signal presented during or after a tape-recorded sentence. The stimulus signal is presented by switching lamps, projectors, noise generators, or similar ac loads, and the latency of the Ss' response is timed with externally triggered electronic stopwatches. In addition, logic circuits for controlling the experimental apparatus must be switched or held during the experiments. This control circuitry also typically includes both ac and de loads.

Since our test materials are presented auditorally, it is imperative that the operation of the timing, presentation, and control circuits produce no disruptive noise or extraneous acoustic cues. Mechanical switches and relays usually introduce such signals by producing either audible clicks or line transients which cause audible interference in audio circuits. Furthermore, the time required to effect mechanical contact closure can vary considerably from trial to trial and as the various components of the relays and switches wear during an experiment. Finally, mechanical switches usually open or close with an arbitrary pattern of pulses which renders the operation of associated logic circuitry unreliable.

We set out to design and build an electronic relay that would not be subject to the bothersome difficulties of mechanical switches and relays and which could readily be adapted to the various needs of our laboratory. To review the requirements: we wanted the relay/switch to change states without producing acoustic or electronic noise; to drive both an ac load (e.g., an incandescent lamp) and a dc load (e.g., an electronic stopwatch) in each state; and to be compatible with existing RTL (resistor-transistor logic) circuits, which operate at $+3.6 \mathrm{~V}$ $\mathrm{dc}$, and TTL (transistor-transistor logic) circuits, which operate at $+5 \mathrm{~V}$ dc.

* This research was supported in part by NIH Grant HD05168-02.
These requirements demand solid-state switching. The fundamental switching operation requires a device that can output two fixed states and switch between them. The JK flip-flop is a device with two logically complementary outputs called $Q$ and $\bar{Q}$. Thus, if $Q$ is in State "HI," $Q$ will be in State "LO," and vice versa. The outputs switch state each time a negative-going transition (HI to $\mathrm{LO}$ ) is applied to the "trigger" (clock) input of the flip-flop. The outputs that follow the trigger input can be predetermined by setting the logic levels of the "set" and "clear" inputs of the flip-flop. We used RTL logic in the switch (with TTL-compatible output), employing a Fairchild 9923 JK flip-flop.

Logic circuitry requires a fairly precise, well-regulated power supply. (The logic diagram is given in Fig. 1.) The upper half of the circuit diagram (Fig. 2) shows the power supply for the switch. The basic component is a Fairchild $\mu \mathrm{A} 723$ precision voltage regulator that can output $2-37 \mathrm{~V}$ dc, regulated negative or positive voltages. The circuit shown produced $3.6 \mathrm{~V} \mathrm{dc}$, and the appropriate equations for computing component values are given in the parts list in Table $1{ }^{1}$ The maximum current output of the 723 is approximately $80 \mathrm{~mA}$. Since our current requirements exceeded this figure, an external series pass transistor (2N4036) was added to boost current capability to about $200 \mathrm{~mA}$. The resistor, $R_{\mathrm{sc}}$, provides short-circuit protection for the 723 .

In operation, the $2 \mathrm{~N} 4036$ transistor overheated due to the large collectoremitter voltage drop $[(12.6 \mathrm{~V} \times 1.4 \mathrm{~V})-3.9 \mathrm{~V}=13.7 \mathrm{~V}]$. The $51-\mathrm{ohm}, 5-\mathrm{W}$ resistor was added to reduce the collector-emitter voltage to about $8 \mathrm{~V}$, at which level the transistor still operated in its linear active region, but no longer overheated. A $10-\mathrm{MHz}$ oscillation appearing at the base of the 2N4036 (probably from the Triacs) was eliminated by adding the $2,000-\mathrm{pF}$ capacitor.

All mechanical switches "bounce" when switched, yielding a waveform like that shown at $S_{1}$ in the circuit diagram. Since the JK flip-flop switches on each negative transition, such a waveform is likely to cause several state changes for each mechanical switching operation. In order that only one state change occur for each stimulus or response, the switch pulse was conditioned so that only one trigger pulse was delivered to the flip-flop each time the switch was operated. The monostable multivibrator (one-shot, or o.s.) shown in the dashed box in the circuit diagram accomplished this conditioning. The duration, $t$, of the pulse was determined by the selection of Capacitor $\mathrm{C}_{2}$, using the formula

$$
t=\mathrm{RC}_{2}=1 \mathrm{~K} \text { ohm } \times \mathrm{C}_{2}=\mathrm{C}_{2} \text { msec }
$$

(where $\mathrm{C}_{2}$ is microfarads).

The one-shot was constructed from half a Fairchild 9914 dual two-input NAND gate and a Fairchild 9900 inverting buffer. The buffer further "squares" the output pulse of the one-shot (RTL flip-flops are extremely sensitive to the rate of HI-LO transition) and has a high output drive factor which insures clean triggering. The $1 \mathrm{~K}-\mathrm{ohm}$ resistor is internal to the 9900.

The trigger pulse width was set at $10 \mathrm{msec}$, long enough to insure that transients from the mechanical switch would not retrigger the one-shot, but short when compared to Ss' reaction times. To determine absolute reaction times, the trigger delay can be added to observed times.

Switching of the ac loads was accomplished by using the Triacs ${ }^{2}$ noted in the circuit diagrams. Sufficient current applied to the gate of a Triac causes it to conduct either + or - voltages. The Triac stops conducting when the current through it is below the holding current and the applied voltage becomes zero. The output drive capability of the flip-flop output was buffered. To guard against the intrusion of noise and to enable the use of 30 - to $60-\mathrm{W}$ loads, an isolation transformer was used to drive the Triacs.

If inductive loads are used, a resistance and capacitance in series must be added across the Triacs. A Triac must turn OFF during the instant when the load current passes through zero. In an inductive circuit, the current lags the voltage and reaches zero after the voltage has attained some value of opposite polarity. Since the Triac tries to open at zero current, and there is no current in the inductor, the instantaneous line voltage will appear across the Triac at a rate $\mathrm{dv} / \mathrm{dt}$, called the commutation $\mathrm{dv} / \mathrm{dt}$. It is the rate of the voltage rise which is impressed on the Triac immediately following turnoff at zero 


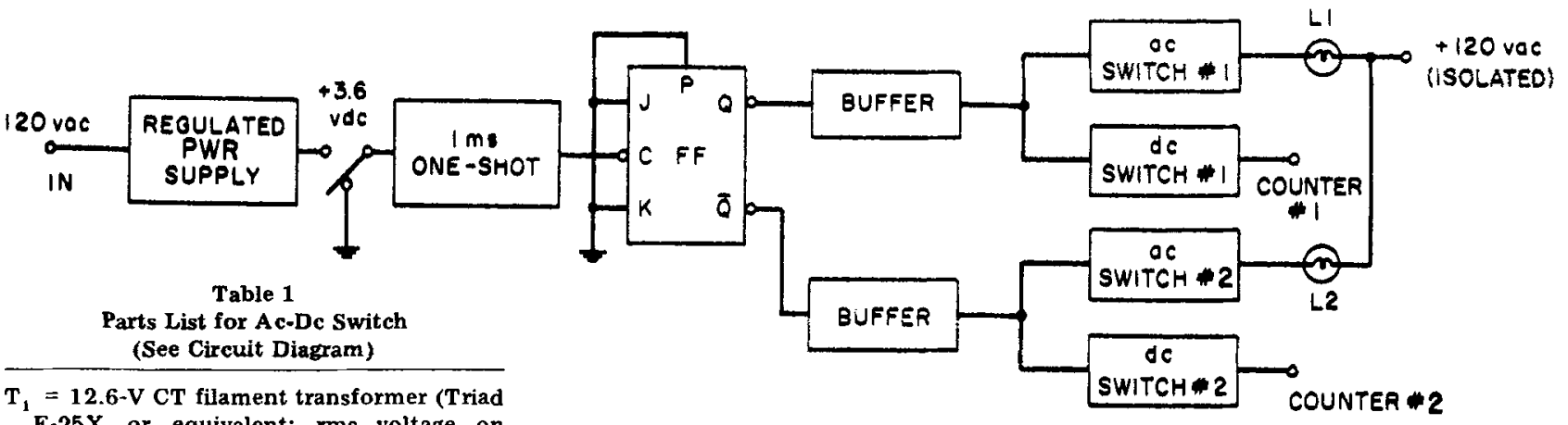

Fig. 1. Logic diagram of relay.

or equivalent: rms voltage on filament must be $\geqslant \mathrm{V}_{\text {ref }}$ on voltage regulator; $V_{\text {ref }}=7.15 \mathrm{~V}$ )

$R_{1}$ is bias for $2 N 4036$ transistor, $I_{c}=$ $500 \mathrm{~mA}$

$C_{1}=2000 \mathrm{pf}$ for elimination of high-frequency $(>1 \mathrm{MHz}$ ) noise

$\mu \mathrm{A723}=$ Fairchild $\mu \mathrm{A723}$ voltage regulator $V_{\text {reg }}=\left(R_{3} / R_{2}+R_{3}\right) V_{\text {ref }}$

$R_{\text {se }}=$ current limiting resistor $R_{\text {sc }}=$ $\mathrm{V}_{\text {reg }} / \mathrm{I}_{\text {lim }}$, where $\mathrm{I}_{\mathrm{lim}}$ is short-circuit current

S1 = any SPDT switch; it can be replaced by optoelectronic switch

oS = one-shot bounceless switch conditioner (monostable multivibrator) pulse width $T=R C$; $T$ is in msec if $R$ is in $K \Omega$ and $C_{2}$ is in $\mu f$. In our case, $T=$ $10 \mathrm{msec}$

$9914=$ Fairchild 9914 dual two-input NAND gate

$9900=$ Fairchild 9900 inverting buffer with internal $1 \mathrm{k} \Omega$ resistor

$\mathrm{FF}=$ Fairchild $9923 \mathrm{JK}$ flip-flop

TR 1, TR $2=200-\mathrm{V} 6 \mathrm{~A}$ triac

$\mathrm{L} 1, \mathrm{~L} 2$ = commercial $30 \mathrm{~W}-60 \mathrm{~W}$ bulb

DC1. DC $2=$ Motorola MFE 3001 n-channel enhancement-mode MOSFET

$\mathrm{T} 2=1: 1$ isolation transformer

Rect = full-wave bridge rectifier $(50 \mathrm{~V}$, $1.5 \mathrm{amp}$ )(Mallory $\mathrm{FW} 50$ or equivalent)

current. The rate is limited only by stray capacitance and the Triac capacitance, Cs.

For the Triac to turn off reliably in an inductive circuit, the rate $\mathrm{dv} / \mathrm{dt}$ must be limited by additional capacitance. Resistance is necessary to damp the ringing of the capacitance with the load inductance and to limit the current surge from the capacitor when the Triac fires.

For almost all inductive loads, $\mathbf{R}_{1}=$ 100 ohms and $C_{1}=0.1$ microF will limit $\mathrm{dv} / \mathrm{dt}$ to $\approx 1 \mathrm{~V} / \mathrm{msec}^{2}$

The dc switching was accomplished with n-channel enhancement-mode MOSFETs (Motorola MFE 3001). ${ }^{3}$ FETs were chosen for their high noise immunity and virtually infinite input impedance, which means that they draw infinitesimal input current. The n-channel enhancement mode MOSFETs were chosen because both the drain and gate operate on positive voltages. The drain resistor
(1.5K ohms) provides sufficient voltage transition to start either RTL or TTL circuits. The + connection of the MOSFETs is attached to the + power supply of our externally triggered timers. The device will switch on any drain supply voltage from +3 to $+20 . \mathrm{V}$ dc and will trigger with as little as $1 \mathrm{~V}$ transition at the gate.

These MOSFETs must be handled with care. Any static charge applied to the substrate can permanently damage the transistor. Consequently, they are supplied with a lead shorting ring which should be removed only after the device is soldered into position. (Further handling instructions are provided with the device.)

The cost of parts is about $\$ 50$. The device would be much less expensive $(\$ 30)$ if it were designed for TTL compatibility alone.

\section{NOTES}

1. Fairchild semiconductor integrated

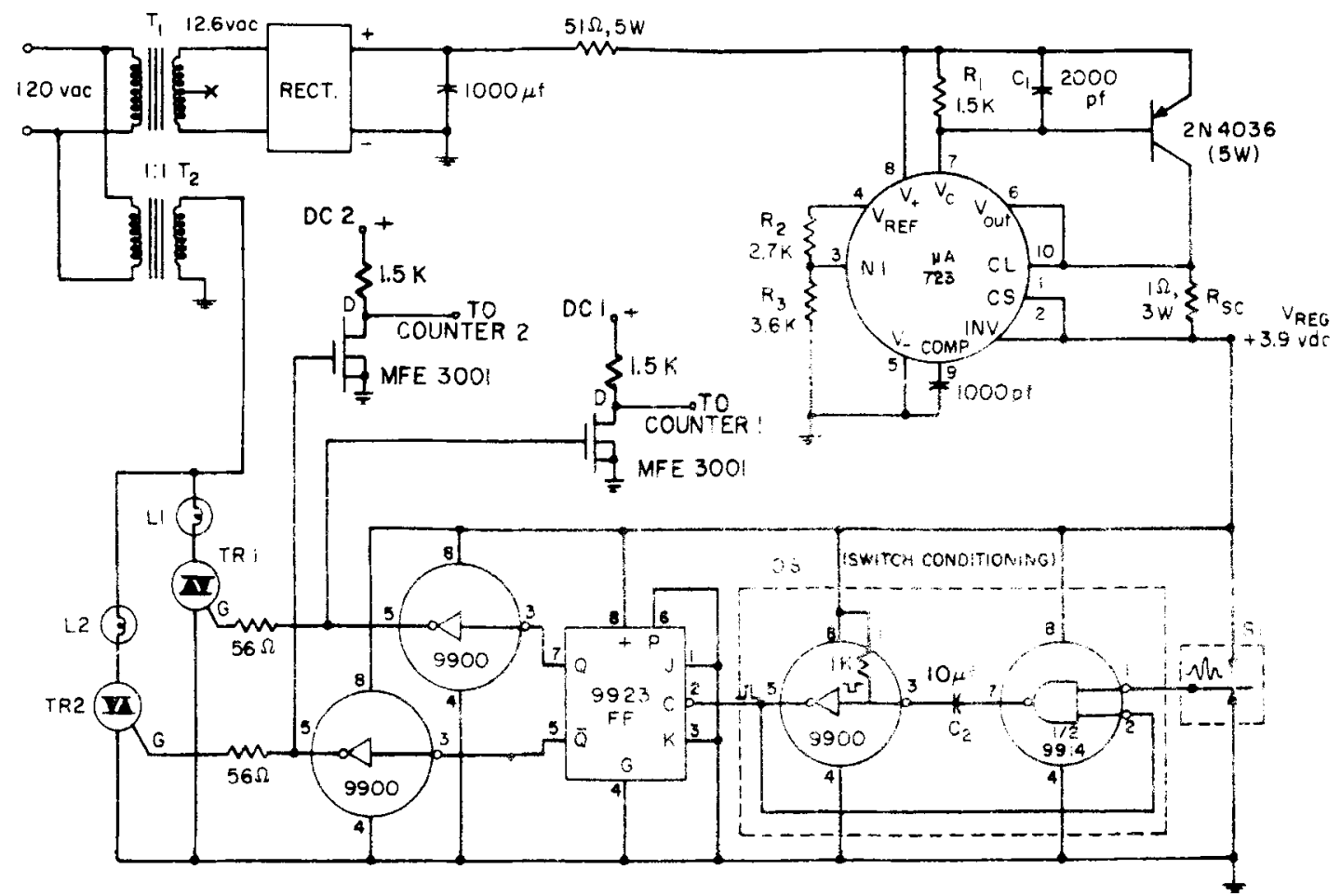

Fig. 2. Circuit diagram of relay. 
circuits data catalog, 1970, Fairchild Semiconductor Company No. BR-BR-0015-29 $100 \mathrm{M}$.

2. General Electric SCR manual, 4 th ed.,
General Electric Products Company, Dept. B., 3800 N. Milwaukee Ave., Chicago, Illinois 60641 .

3. Motorola semiconductor data book,
Motorola Semiconductor Products Division

Technical Information Center, P.o. Box 95, Phoenix, Arizona 85001. 